\title{
Metastatic parathyroid carcinoma treated with radiofrequency ablation: A novel therapeutic modality
}

\author{
Riddhi DasGupta1 ${ }^{1}$, Sahana Shetty ${ }^{1}$, Shyamkumar Nidugala Keshava ${ }^{2}$, Mayank Gupta ${ }^{3}$,
}

Mazhuvanchary Jacob Paul ${ }^{4}$, Nihal Thomas ${ }^{1}$

1. Department of Endocrinology, Diabetes and Metabolism, Christian Medical College, Vellore, India

2. Department of Radiology, Christian Medical College, Vellore, India

3. Department of Pathology, Christian Medical College, Vellore, India

4. Department of Endocrine Surgery, Christian Medical College, Vellore, India

\section{CASE STUDY}

Please cite this paper as: DasGupta R, Shetty S, Keshava SN, Gupta M, Paul MJ, Thomas N. Metastatic parathyroid carcinoma treated with radiofrequency ablation: A novel therapeutic modality. AMJ 2014;7(9):372-375. http://doi.org/10.21767/AMJ.2014.2084

Corresponding Author:

Dr Nihal Thomas

Department of Endocrinology, Diabetes and Metabolism, Christian Medical College, Vellore, India

Email: nihal_thomas@yahoo.com

\section{ABSTRACT}

Parathyroid carcinoma (PCA), accounting for less than one per cent of all endocrine malignancies, is a rare cause of primary hyperparathyroidism. A diagnosis of parathyroid carcinoma may be challenging in the presence of localised disease and involves a histological diagnosis based on capsular, vascular, or perineural invasion or the presence of metastasis. Distant metastasis remains a rare presentation, with the lung being the most common site. Surgery remains the treatment of choice as radiotherapy and chemotherapy have proved to be of limited benefit in metastatic disease. This case reports suggests that radiofrequency ablation has the potential to be a novel and effective treatment option in these patients.

\section{Key Words}

Parathyroid, carcinoma, radiofrequency, metastasis

\section{Implications for Practice:}

\section{What is known about this subject?}

Parathyroid carcinoma is a rare endocrine malignancy wit $h$ an incidence of 5.73 per 10 million persons.

\section{What new information is offered in this case study?}

Metastatic parathyroid carcinoma was successfully treate $d$ using radiofrequency ablation.

\section{What are the implications for research, policy, 0}

\section{practice?}

Radiofrequency ablation has the potential to be a novel an $d$ effective alternative to surgery in severe metastatic disease.

\section{Background}

Parathyroid carcinoma (PCA) is a rare malignant neoplas $n$ derived from the parenchymal cells of the parathyroid glands. Patients with PCA typically have severe primary hyperparathyroidism (PHPT) with marked hypocalcaemia $(14 \mathrm{mg} / \mathrm{dl})$ and profoundly elevated (three to 10 times normal) parathyroid hormone (PTH) levels. These patients are more likely to have metabolic bone and renal sequelae than those with benign parathyroid adenomas. Parathyroid carcinoma with metastasis significantly worsens the overall disease prognosis.

The diagnosis of PCA remains challenging in light of the absence of definite histopathological markers. Local invasion, lymph node involvement or distant metastases remain the mainstay of diagnosis, although a few authors like Obara et al. have suggested certain ultrasound features which may provide a diagnostic clue. ${ }^{1}$ Although en bloc surgery-involving removal of the entire parathyroid gland and the capsule around it, the contiguous lymph nodes, half of the thyroid gland on the same side of the body as the cancer along with the adjacent muscles, tissues, and nerves -is the primary modality of treatment, metastatic disease often results in a resistant and potentially fatal form 
of severe hyperparathyroidism. Chemotherapy and local radiation therapy have been met with conflicting results. The rarity of the disease and lack of definite treatment guidelines has contributed to a therapeutic conundrum. As such, radiofrequency ablation of functioning metastatic lesions may provide an intriguing and effective treatment alternative for this extremely rare and challenging disease. We report a case of parathyroid carcinoma with pulmonary metastasis treated with radiofrequency ablation in a 48year-old South Indian male.

\section{Case details}

A 48-year-old man, a patient known to have primary hyperparathyroidism for 13 years with a history of recurrent fractures and renal stones, presented to us with severe generalised bony pain and restricted mobility. His symptoms began 13 years previously when he had severe left-sided hip pain and difficulty with movement. He was found to have biochemical evidence of primary hyperparathyroidism with a left-sided iliac giant cell tumour. The patient underwent resection of the left iliac tumour along with a right inferior parathyroidectomy. Following the surgery, he went into clinical and biochemical remission and was asymptomatic for the next 10 years.

He suffered a left femoral fracture one year ago. On reevaluation at another centre, he was detected to have primary hyperparathyroidism with a right superior parathyroid adenoma on imaging. He was also found to have a right ureteric calculus. He underwent a right superior parathyroidectomy, however, postoperatively his clinical and biochemical parameters failed to improve. He was known to be hypertensive and had undergone coronary artery bypass grafting for triple vessel coronary artery disease six months prior to admission to our institution and incurred another fracture of the right femur two months prior to his current admission. There was no significant family history.

At presentation, the patient complained of symptoms of severe bony pain, restricted mobility, and generalised body aches. Investigations revealed: corrected serum calcium of $12.8 \mathrm{mg} / \mathrm{dl}(8.5-10.0 \mathrm{mg} / \mathrm{dl})$, phosphate of $1.7 \mathrm{mg} / \mathrm{dl}(2.5-$ $4.0 \mathrm{mg} / \mathrm{dl})$, parathormone (PTH) levels of $1,500 \mathrm{pg} / \mathrm{ml}(8.0-$ $52.0 \mathrm{pg} / \mathrm{ml})$, and creatinine of $0.67 \mathrm{mg} / \mathrm{dl}(0.6-1.5 \mathrm{mg} / \mathrm{dl})$. Radiological investigations revealed fractures of both femurs with in situ dynamic hip screw (DHS) implants. Dualenergy $x$-ray absorptiometry scans showed severe osteoporosis of the lumbar spine, femoral neck, and forearm. An ultrasound abdomen revealed bilateral medullary nephrocalcinosis with a small left renal calculus.
The parathyroid scintigraphy did not reveal any parathyroid adenoma. He subsequently underwent an F-18 fluorodeoxyglucose-PET (positron emission tomography) scan, which revealed hypermetabolic foci suggestive of metastasis in bilateral lung lobes, along with extensive lytic lesions suggestive of giant cell tumours involving the skull, vertebrae, scapula, humeri, and pelvic bones.

A corresponding contrast enhanced computerised tomography scan (CECT) of the thorax was suggestive of two well-defined lung nodules measuring $25 \times 23 \mathrm{~mm}$ in the right upper lobe and $29 \times 36 \mathrm{~mm}$ in the right lower lobe. A CTguided biopsy from the pulmonary lesions was subjected to a histopathological examination along with tissue parathormone (PTH) assay from the biopsy sample. The histopathological picture was suggestive of parathyroid carcinoma with the corresponding PTH levels from the tissue samples being $1,800 \mathrm{pg} / \mathrm{ml}$ (Figure 1). A diagnosis of primary parathyroid carcinoma with functioning pulmonary metastasis with severe hyperparathyroidism was considered in view of the clinical and investigation profile.

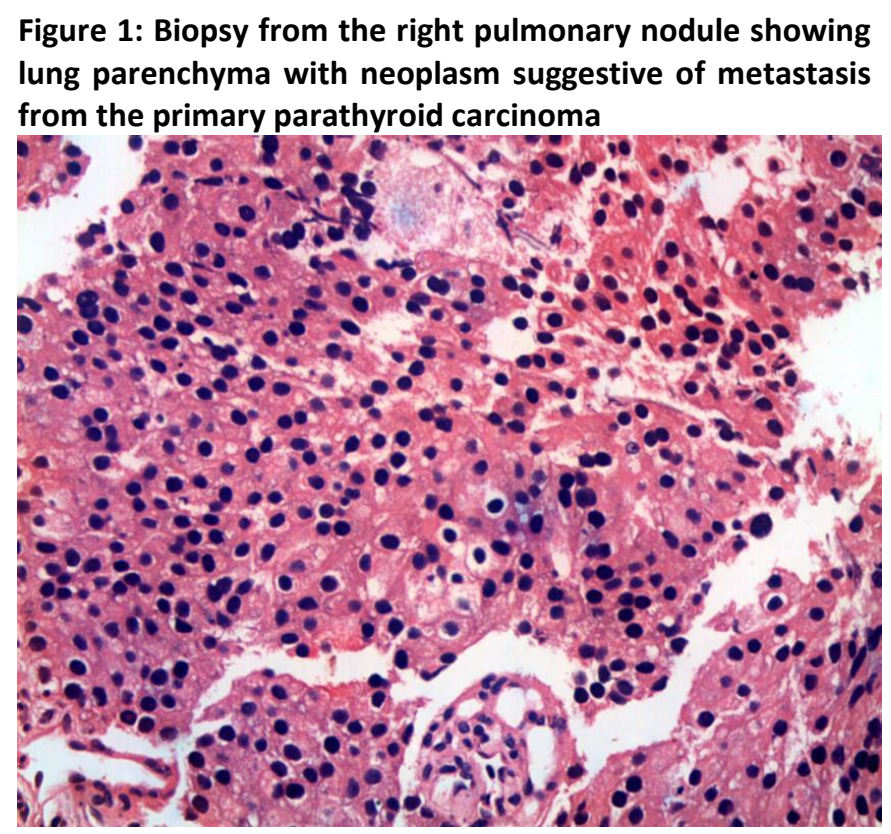

The treatment options that were initially considered included surgical resection of the metastatic lung nodules, radiotherapy to the lung lesions, and chemotherapy. Owing to the multiplicity of metastatic lesions, severe metabolic bone disease, the need for extensive lung resection and cardiac comorbidities, none of the existing treatment options were tenable. Hence, radiofrequency ablation of the pulmonary nodules was considered as the most viable alternative in the background of this difficult clinical scenario. A review of existing literature showed case studies from Japan ${ }^{6}$ and Brazil, ${ }^{7}$ which have used radiofrequency 
ablation treatment for metastatic parathyroid carcinoma, although the numbers were few. The procedure was undertaken by interventional radiologists (Figures $2 a, 2 b$, and 2 c) and was well tolerated by the patient.

Post-procedure there was a dramatic improvement in the patient's clinical symptoms and biochemical parameters; within 72 hours post-ablation, the corrected serum calcium dropped to $9.5 \mathrm{mg} / \mathrm{dl}$, serum phosphate improved to $2.7 \mathrm{mg} / \mathrm{dl}$, and serum PTH (iced) levels had declined to $400 \mathrm{pg} / \mathrm{dl}$. The patient had significant improvement in his functional ability and was stable at the three months followup.

Figure 2a: RFA needle inserted in the centre of the nodule using robotic guidance device Maxio and CT scan

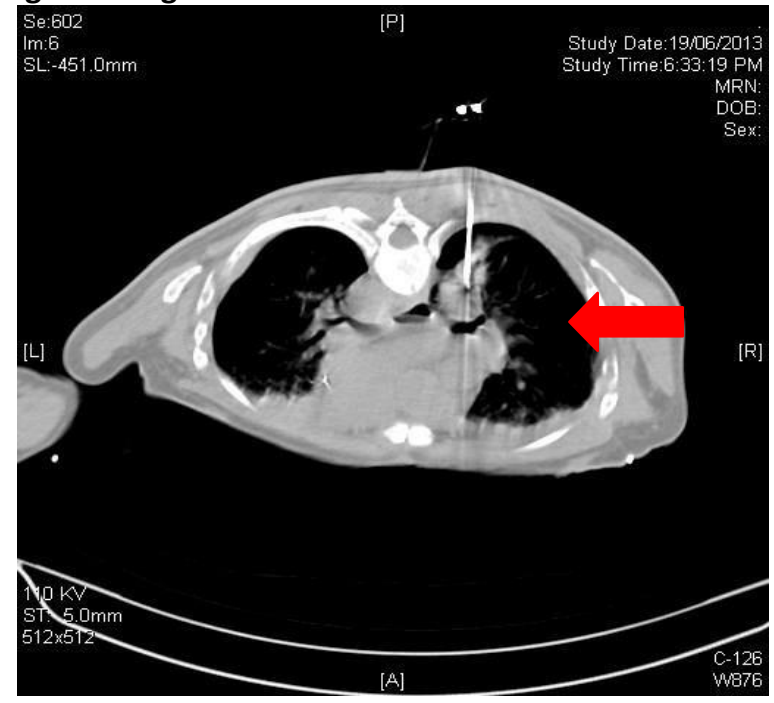

Figure $2 \mathrm{~b}$ : The tines of the needle were heated up to $90^{\circ} \mathrm{C}$ leading to gradual ablation of the pulmonary nodule with progressive shrinkage

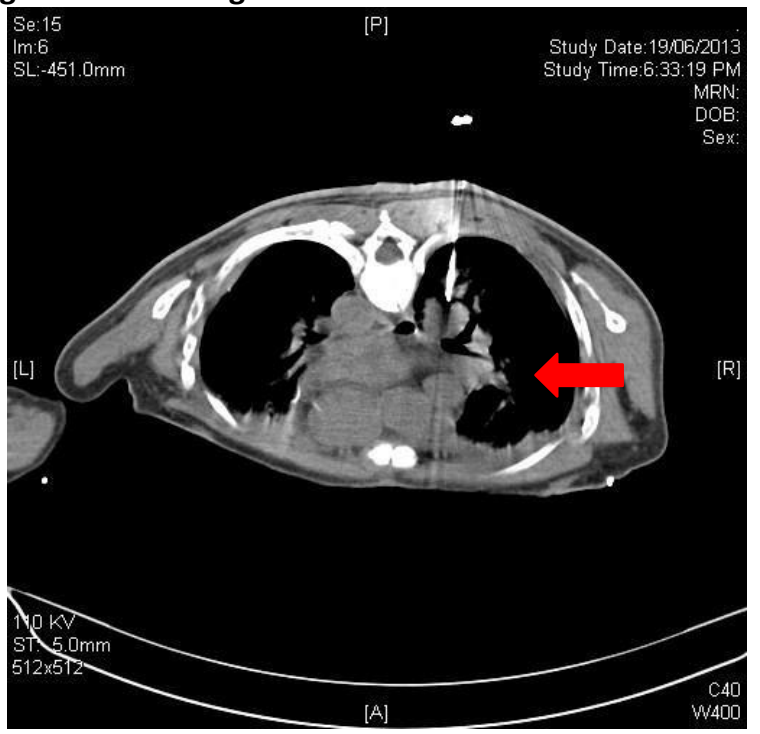

Figure 2c: The needle was maintained for $\mathbf{2 2}$ minutes with complete ablation of the lesion leading to a reduction in the size of the lesion

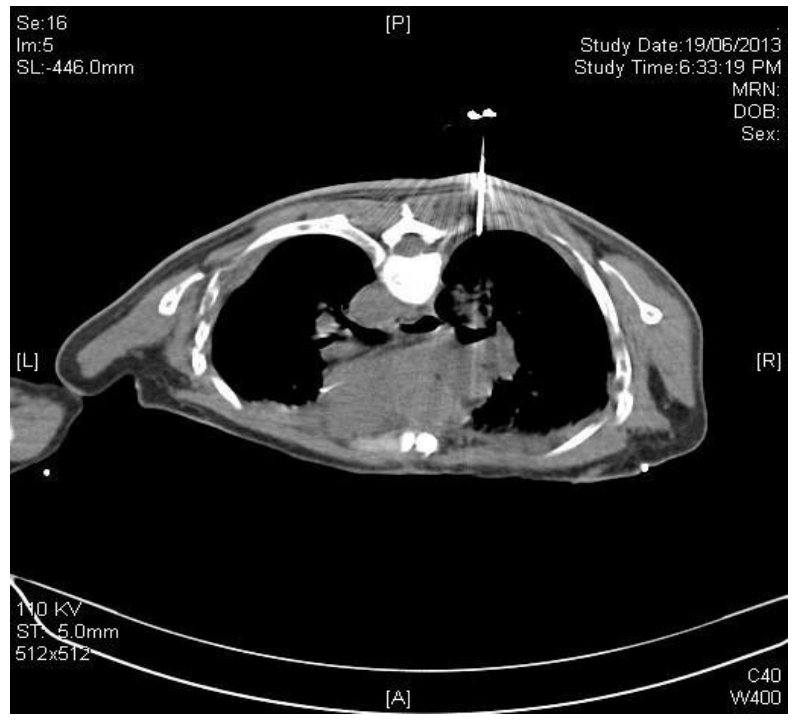

\section{Discussion}

Parathyroid carcinoma (PCA) is a rare disease accounting for 0.5 per cent of all malignancies and less than 2 per cent of primary hyperparathyroidism (HPT) cases. ${ }^{2}$ HRPT2 gene mutations play a central role in the pathogenesis of parathyroid carcinoma and are observed not only in sporadic but also in familial cases. Reports of lung metastases from parathyroid carcinoma are very rare. It is challenging to treat patients with functioning lung metastases from parathyroid carcinoma. Systemic therapy has minimal effects on the course of the disease and the hypocalcaemia that coexists.

Resection of pulmonary metastases is one of the therapeutic options. Obara et al. ${ }^{3}$ reported that 50 per cent of patients with pulmonary metastases achieved successful palliation and/or long-term survival with repeated thoracotomy. En bloc resection as opposed to simple parathyroidectomy is associated with a significantly lower rate of recurrence and greater survival benefit. ${ }^{4}$ Unfortunately, not all patients are surgical candidates and alternative modalities are limited. PCA is not a radiosensitive neoplasm and in most patients studied, radiotherapy failed to slow the tumour growth and reduce hormonal secretion. ${ }^{5}$ Cytotoxic chemotherapy with various regimens involving doxorubicin, cyclophosphamide, and 5 fluorouracil has been ineffective in treating PCA. ${ }^{6}$

Recent experience with radiofrequency ablation of metastatic PCA has been shown to control disease in Japan ${ }^{7}$ and $\mathrm{Brazil}^{8}$ with survival benefits of up to 15 months. We report the first instance of use of radiofrequency ablation in a case of parathyroid carcinoma with functioning lung 
metastasis from India with a significant impact on the disease outcome. In view of the rarity of the disease and challenges with regard to diagnosis and management of parathyroid carcinoma, attempts to evolve newer therapeutic modalities to treat the disease are essential.

\section{Conclusion}

PCA can be difficult to treat and the disease may not respond to many of the common interventions. This single case provides an example of how radiofrequency ablation can provide a novel and useful adjunct to established treatment for metastatic parathyroid carcinoma. Clinicians should be aware that radiofrequency ablation might be a potential option if other PCA interventions have had limited success in treatment of the disease.

\section{References}

1. Obara $T$, Okamoto $T$, Ito $\mathrm{Y}$, Yamashita $\mathrm{T}$, Kawano $\mathrm{M}$, Nishi T, Tani M, Sato K, Demura H, Fujimoto Y. Surgical and medical management of patients with pulmonary metastasis from parathyroid carcinoma. Surgery. 1993 Dec;114(6):1040-8; discussion 1048-9.

2. Shane E. Clinical review 122: Parathyroid carcinoma. J Clin Endocrinol Metab. 2001 Feb;86(2):485-93.

3. Obara T, Fujimoto Y. Diagnosis and treatment of patients with parathyroid carcinoma: An update and review. World J Surg. 1991 Nov-Dec;15(6):738-44.

4. Cohn K, Silverman M, Corrado J, Sedgewick C. Parathyroid carcinoma: The Lahey Clinic experience. Surgery 1985;98:1095-1100.

5. Anderson BJ, Samaan NA, Vassilopoulou-Sellin R, Ordonez NG, Hickey RC. Parathyroid carcinoma: Features and difficulties in diagnosis and management. Surgery. 1985 Dec;98(6):1095-100.

6. Wiseman SM, Rigual NR, Hicks WL Jr, Popat SR, Lore JM Jr, Douglas WG, Jacobson MJ, Tan D, Loree TR. Parathyroid carcinoma: A multicenter review of clinicopathologic features and treatment outcomes. Ear Nose Throat J. 2004 Jul;83(7):491-4.

7. Iguchi T, Yasui K, Hiraki T, Gobara H, Mimura H, Sato S, Fujiwara H, Yano A, Doihara H, Kanazawa $S$. Radiofrequency Ablation of Functioning Lung Metastases from Parathyroid Carcinoma. J Vasc Interv Radiol. 2008 Mar;19(3):462-4.

8. Delmar EL, Hoff OA, Alcantara AEE, Martins RM, Correa PHS, Menezes M. Radiofrequency Ablation of Pulmonary Metastases in Parathyroid Carcinoma: An Alternative Therapy for Severe Refractory Hypercalcemia. Paper presented at The Endocrine Society's 94th Annual Meeting and Expo; 2012 June 23-26, 2012; Houston, USA.

\section{PEER REVIEW}

Not commissioned. Externally peer reviewed.

\section{CONFLICTS OF INTEREST}

The authors declare that they have no competing interests.

\section{PATIENT CONSENT}

The authors, DasGupta R, Shetty S, Keshava SN, Gupta M, Paul MJ, Thomas N, declare that:

1. They have obtained written, informed consent for the publication of the details relating to the patient(s) in this report.

2. All possible steps have been taken to safeguard the identity of the patient(s)

3. This submission is compliant with the requirements of local research ethics committees. 